\title{
Adaptive Sliding Mode Control Method Based on Nonlinear Integral Sliding Surface for Agricultural Vehicle Steering Control
}

\author{
Taochang $\mathrm{Li}^{1,2}$ and Jingtao $\mathrm{Hu}^{1,2}$ \\ ${ }^{1}$ Shenyang Institute of Automation, Chinese Academy of Sciences, Shenyang 110016, China \\ ${ }^{2}$ University of Chinese Academy of Sciences, Beijing 100049, China \\ Correspondence should be addressed to Taochang Li; litaochang@163.com
}

Received 26 November 2013; Revised 26 February 2014; Accepted 27 February 2014; Published 30 March 2014

Academic Editor: Anuar Ishak

Copyright (C) 2014 T. Li and J. Hu. This is an open access article distributed under the Creative Commons Attribution License, which permits unrestricted use, distribution, and reproduction in any medium, provided the original work is properly cited.

\begin{abstract}
Automatic steering control is the key factor and essential condition in the realization of the automatic navigation control of agricultural vehicles. In order to get satisfactory steering control performance, an adaptive sliding mode control method based on a nonlinear integral sliding surface is proposed in this paper for agricultural vehicle steering control. First, the vehicle steering system is modeled as a second-order mathematic model; the system uncertainties and unmodeled dynamics as well as the external disturbances are regarded as the equivalent disturbances satisfying a certain boundary. Second, a transient process of the desired system response is constructed in each navigation control period. Based on the transient process, a nonlinear integral sliding surface is designed. Then the corresponding sliding mode control law is proposed to guarantee the fast response characteristics with no overshoot in the closed-loop steering control system. Meanwhile, the switching gain of sliding mode control is adaptively adjusted to alleviate the control input chattering by using the fuzzy control method. Finally, the effectiveness and the superiority of the proposed method are verified by a series of simulation and actual steering control experiments.
\end{abstract}

\section{Introduction}

Automatic steering control is the base and one of the key technologies to achieve agricultural vehicle automatic navigation control and its control performance directly influences the navigation control accuracy. Therefore, it is necessary to carry out the research on automatic steering control of agricultural vehicles.

There are mainly two approaches to drive an agricultural vehicle automatic steering control system. One is to utilize a motor to drive the steering wheel such that the steering angles are changed indirectly $[1,2]$. The other is to install an electrohydraulic steering control loop which is attached to the original hydraulic steering system, such that the hydraulic steering circuit is controlled directly $[3,4]$.

For the first driving approach of the automatic steering control system, Zhang [5] and Lian [6], respectively, develop PID steering control methods based on permanent magnet brushless DC motor driving mechanisms and obtain satisfactory results by adjusting the PID gains when the disturbances are relatively small. Zhou [7] and Gao et al. [8] design agricultural vehicle steering driving systems based on step motors and, respectively, utilize the Bang-Bang control method and the PID method to develop the steering controllers of agricultural vehicles. For the second driving approach, Zhang et al. develop automatic steering controllers based on the fuzzy control method for agricultural vehicles, and the control performances are verified by the practical steering control experiments [9-12]. Some researchers also use the PID method to design the steering controllers for the steering system which is implemented by the second driving approach $[13,14]$. In order to improve the control precision of the PID controllers, a cascade closed-loop PID control system is designed and then the control precision is improved, but the measurement of the steering angular velocity is difficult [15]. Practice has shown that the PID method and the fuzzy control method are the simplest and the most straightforward choices when the steering system 
mathematic model is unknown. From the perspective of model-based control, since the sliding mode control method is an effective approach for the control of the dynamical systems with uncertainties [16-18], some researchers also deduce the nominal mathematic model of the agricultural vehicle steering system and regard the uncertainties of the system as equivalent disturbances. Then an automatic steering controller is designed based on the sliding mode control method and the robustness of the closed-loop control system is guaranteed when the system suffers from the disturbances [19]. However, the main drawback of the sliding mode control is that the control input chattering is large when the defined disturbance bounds are large [20]. Although some adaptive sliding mode control methods are developed for alleviating chattering phenomena of the control input in [21-24], none of the above methods can solve the conflict between the system response time and the system output overshoot.

To answer the abovementioned questions, a novel adaptive sliding mode control method based on a nonlinear integral sliding surface is proposed in the paper for agricultural vehicle automatic steering control. The method can guarantee the prompt and nonovershoot response characteristics of the steering control system and effectively alleviate the control input chattering phenomenon arising in the traditional sliding mode control when the steering system suffers from disturbances. The remainder of the paper is divided into five sections. In Section 2, problem statement for the agricultural vehicle steering control is presented. In Section 3, the adaptive sliding mode control method based on nonlinear integral sliding surface is proposed. Then, the feasibility and the superiority of the proposed method are validated by the simulation results and the experimental results in Sections 4 and 5, respectively. Finally, conclusions are drawn in Section 6.

\section{Problem Statement for Agricultural Vehicle Steering Control}

A typical automatic navigation control system for agricultural vehicles mainly includes a navigation controller, a steering controller, position and orientation sensors, a steering angle sensor, and a steering actuator. The schematic diagram of the automatic navigation control system is shown in Figure 1.

The navigation controller decides desired steering angle commands according to the current position and orientation of the vehicle as well as the path information and then sends the desired angles to the steering controller. The steering controller determines the control inputs of the steering actuator to drive the vehicle steering system and guarantees the actual steering angles to track the desired steering angles quickly. Finally, the automatic navigation control for agricultural vehicles is achieved. From the above description, it can be concluded that the automatic steering control is the key and the prerequisite to achieve automatic navigation control and its control performance will directly influence the navigation control accuracy. In the paper, it is assumed that the highlevel navigation controller has already determined the desired steering angle command to maintain the vehicle on the path.
It is left to a low-level steering controller to ensure that the actual steering angle tracks the desired steering command.

Only the automatic steering control problem is studied in the paper, so it is assumed that there has already been constant steering angle command $\delta_{d}$ in each navigation control period. Subsequently, a steering control method is needed to determine the control inputs $u$ of the steering actuator which drives the steering wheels to track the desired steering angles $\delta_{d}$. The ultimate objective is to realize the desired steering actions accurately and promptly according to the desired steering angle commands when the steering system suffers from uncertain disturbances.

Since the electrohydraulic $(\mathrm{E} / \mathrm{H})$ steering actuator has the capability of quick response and precise execution, it is widely used in high precision navigation control systems of agricultural vehicles [25]. In view of this, the electrohydraulic steering actuator is also designed in the paper to achieve the automatic steering control system. Specifically, an electronic proportional valve and three solenoid valves are installed on the original hydraulic steering system of the agricultural vehicles (such as tractors). The automatic steering controller controls the electronic proportional valve spool position to regulate the hydraulic oil flow rate passing through the valve. Consequently, the velocity of the steering cylinder rod is changed and then the steering angles are also regulated. The solenoid valves achieve the manual and automatic switching of the steering hydraulic oil circuit. In addition, since the driving current of the electronic proportional valve is very large, a current amplifier is also chosen to drive the valve.

The principle of the automatic steering system for agricultural vehicle navigation control is shown in Figure 2. In the figure, $\delta_{d}$ denotes the desired steering angle, $\delta$ stands for the actual steering angle, $i$ denotes the control current of the proportional valve, and $Q$ is the flow rate of the proportional valve. In the paper, the current amplifier, the electronic proportional valve, and the solenoid valves are collectively referred to as the steering actuator.

In order to design the complete automatic steering system and an effective control strategy for it, it is necessary to establish the mathematic model of the plant in Figure 2. According to [26], it is reasonable to model the plant as a second-order model, as indicated by transfer function

$$
\frac{\delta(s)}{u(s)}=\frac{k_{g}}{s(\tau s+1)},
$$

where $\delta(s)$ is steering angle, $u(s)$ is control current, $k_{g}$ is $\mathrm{E} / \mathrm{H}$ steering system gain, and $\tau$ is $\mathrm{E} / \mathrm{H}$ steering system time constant.

Let $x_{1}=\delta$ and $x_{2}=\dot{\delta}$; then the transfer function (1) can be converted into the state space model which consists of the state equation (2) and the output equation (3).

State Equation. Consider

$$
\begin{aligned}
& \dot{x}_{1}=x_{2} \\
& \dot{x}_{2}=\frac{-1}{\tau} x_{2}+\frac{k_{g}}{\tau} u .
\end{aligned}
$$




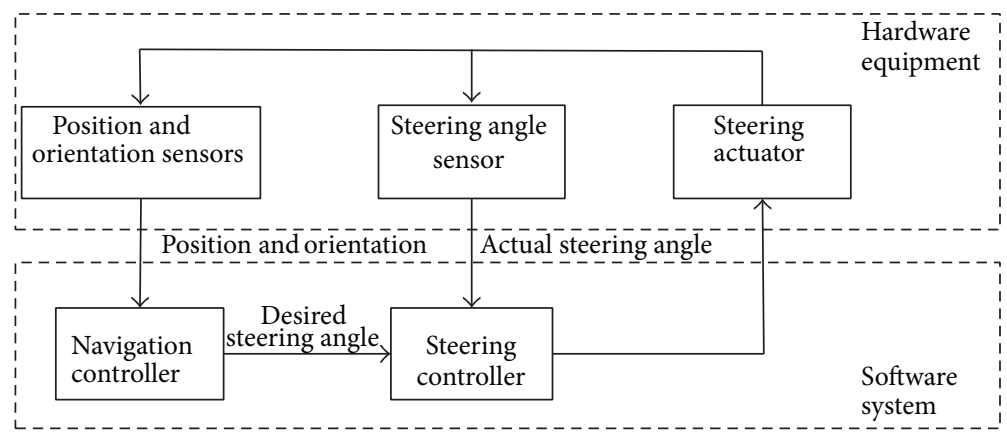

FIGURE 1: The schematic diagram of agricultural vehicle automatic navigation control system.

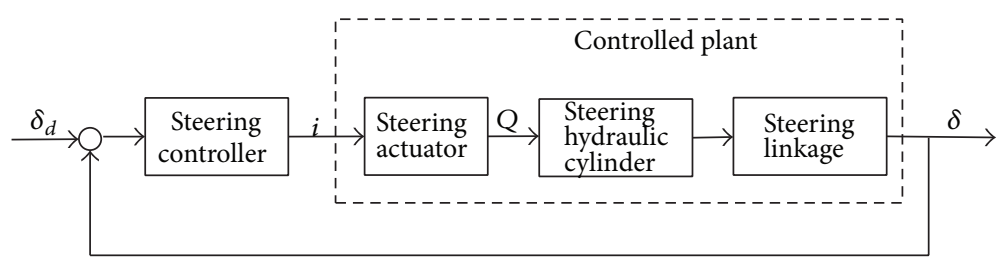

Figure 2: The principle diagram of agricultural vehicle steering control.

Output Equation. Consider

$$
y=x_{1}
$$

As there are unmodeled high frequency dynamics, nonlinear characteristics, and the external disturbances such as the interaction uncertainties between tires and road surface, the $\mathrm{E} / \mathrm{H}$ steering system is an uncertain system. These uncertain factors are regarded in the paper as the disturbances $d(x)$ satisfying a certain bound. Hence, the E/H steering system state equation can be described by

$$
\begin{aligned}
& \dot{x}_{1}=x_{2}, \\
& \dot{x}_{2}=-a x_{2}+k u+d(x),
\end{aligned}
$$

where $a=1 / \tau, k=k_{g} / \tau$.

When there are model parameter variations, the state equation (4) can be expressed as

$$
\begin{aligned}
& \dot{x}_{1}=x_{2}, \\
& \dot{x}_{2}=-\left(a_{0}+\Delta a\right) x_{2}+\left(k_{0}+\Delta k\right) u+d(x),
\end{aligned}
$$

where $k_{0}$ and $a_{0}$ are nominal parameters of the system and $\Delta k$ and $\Delta a$ are parameters perturbation of the system.

Further, state equation (5) can be rewritten as

$$
\begin{aligned}
& \dot{x}_{1}=x_{2}, \\
& \dot{x}_{2}=-a_{0} x_{2}+k_{0} u+D(x),
\end{aligned}
$$

where $D(x)=d(x)-\Delta a \cdot x_{2}+\Delta k \cdot u$ is the equivalent disturbance.

Since the equivalent disturbance $D(x)$ involves many factors and variables, it is difficult to determine a constant upper-bound $\Gamma$ of disturbance and to ensure that $\Gamma$ is small enough for all cases of the system. In order to guarantee the stability of the robust control system, a big value of $\Gamma$ must be used. However, it will lead to the control force obtained being too big. Therefore, in order to solve this problem, the fuzzy control method is adopted in the paper to estimate the disturbance bound $\Gamma$.

\section{Adaptive Sliding Mode Control Method Based on Nonlinear Integral Sliding Surface}

The sliding mode control (SMC) design approach consists of two steps. First, a sliding surface is designed such that the system trajectory along the surface acquires certain desired properties. Then, a discontinuous control law is deduced to ensure that the system trajectories reach the surface in finite time $[27,28]$.

The ideal goal of agricultural vehicle steering control is to make the actual steering angle to track the desired steering angle promptly and accurately when the steering system suffers from the equivalent disturbances. It is well known that a quick response may be obtained at the cost of a large overshoot in most control design schemes. In order to obtain the quick and nonovershoot response characteristic of the steering system, a nonlinear integral sliding surface is proposed in the paper. The sliding surface not only has the advantage of an ordinary integral sliding surface but also has monotonously increasing damping ratio characteristic. It is the characteristic that guarantees, on the one hand, that the closed-loop steering system has relatively smaller damping ratio to accelerate the output response speed during the initial phase of the sliding mode movement and, on the other hand, that the closed-loop steering system has relatively bigger damping ratio to avoid system output overshoot and ensure the system stability as the output approaches the desired 
command. Subsequently, the sliding mode control law is deduced according to the designed sliding surface and the switching gain in the control law is also adaptively adjusted to alleviate the control input chattering by using the fuzzy control method.

3.1. Nonlinear Integral Sliding Surface. The desired steering angle command value of the steering control system is constant during each navigation control cycle. In order to avoid the jump of the command value, it is necessary to construct a transient process such that the output of the system can reasonably follow. The benefit to do this is that we can decrease the initial error of the system to avoid big initial shock force to the steering actuator subsystem. The transient process must meet the following requirements:

(a) meet the executing capacity of the steering system;

(b) reach the command value of the desired steering angle smoothly during the transient process time.

According to requirements (a) and (b), function (7) can be utilized to construct the transient process of the steering control system. Consider

$$
\begin{aligned}
& h(T, t) \\
& = \begin{cases}\frac{\delta_{d}-\delta_{p}}{2}\left(1+\sin \left(\pi\left(\frac{t}{T}-\frac{1}{2}\right)\right)\right)+\delta_{p} & (t \leq T), \\
\delta_{d} & (t>T),\end{cases}
\end{aligned}
$$

where $\delta_{d}$ is the current desired steering angle, $\delta_{p}$ is the previous desired steering angle, and $T$ is transient process time.

Then, the first-order differential expression of the transient process is as follows:

$$
\frac{d^{(1)} h(T, t)}{d t}= \begin{cases}\frac{\left(\delta_{d}-\delta_{p}\right) \pi}{2 T} \cos \left(\pi\left(\frac{t}{T}-\frac{1}{2}\right)\right) & (t \leq T), \\ 0 & (t>T) .\end{cases}
$$

Similarly, its higher order differential expression can also be obtained. The general definition of the differential expression is as follows:

$$
d^{(i)} h:=\frac{d^{(i)} h(T, t)}{d t},
$$

where $i$ is the order of the differential expression.

Based on the transient process and its derivative, the steering angle tracking error and its derivative are defined as $e_{1}=\delta-h(T, t)=x_{1}-h(T, t)$ and $e_{2}=\dot{\delta}-d^{(1)} h=x_{2}-d^{(1)} h$.

Subsequently, a nonlinear integral sliding surface is developed in the paper as follows:

$$
\begin{aligned}
s= & c_{1} \int_{0}^{t}\left(x_{1}-h(T, t)\right) d t+\left(c_{2}-g\right)\left(x_{1}-h(T, t)\right) \\
& +\left(x_{2}-d^{(1)} h\right) \\
= & c_{1} \int_{0}^{t} e_{1} d t+\left(c_{2}-g\right) e_{1}+e_{2},
\end{aligned}
$$

where $c_{1}$ and $c_{2}$ are positive real numbers, and

$$
g=\frac{\lambda}{e^{-1}-1}\left(e^{\left(\left(y-\delta_{p}\right) /\left(\delta_{d}-\delta_{p}\right)\right)^{2}-1}-e^{-1}\right),
$$

where $\lambda$ is an adjustable positive parameter, and $g \in$ $\left[\begin{array}{ll}-\lambda & 0\end{array}\right]$.

Theorem 1. If the nonlinear integral sliding surface (10) is utilized in the SMC, then during the sliding mode movement phase the closed-loop control system is stable and has variable damping ratio $\zeta=\left(c_{2}-g\right) / 2 \sqrt{c_{1}}$ which gradually increases as the system output approaches the desired command.

Proof. According to the nonlinear integral sliding surface (10) and the steering system model (6), the system error model during the sliding mode movement phase is obtained as follows:

$$
\ddot{e}_{1}+\left(c_{2}-g\right) \dot{e}_{1}+c_{1} e_{1}=0 .
$$

Then, the characteristic equation of the closed-loop system is

$$
s^{2}+\left(c_{2}-g\right) s+c_{1}=0 .
$$

Since $c_{1}>0, c_{2}>0$, and $g \leq 0$, the coefficients of the characteristic equation are positive.

Hence, the closed-loop control system is stable according to Hurwitz stability criterion [29].

According to characteristic equation (13), the damping ratio of the closed-loop control system can be deduced as (14) during the sliding mode movement phase. Consider

$$
\zeta=\frac{c_{2}-g}{2 \sqrt{c_{1}}} .
$$

Since $g$ is a monotonically decreasing function with respect to the system output, according to formula (14), it can be deduced that $\zeta$ gradually increases as the system output approaches the desired command.

According to Theorem 1, the closed-loop control system not only is stable but also has variable damping ratio $\zeta \epsilon$ $\left[\zeta_{\min }, \zeta_{\max }\right]$ by using the designed nonlinear integral sliding surface (10). Therefore, we can acquire the desired closedloop system properties that the closed-loop steering system has relatively smaller damping ratio to accelerate the output response speed during the initial phase of the sliding mode movement and has relatively bigger damping ratio to avoid system output overshoot and ensure the system stability as the output approaches the desired command.

According to value domain of the nonlinear function $g$, it can be found that

$$
\begin{gathered}
\zeta_{\min }=\frac{c_{2}}{2 \sqrt{c_{1}}}, \\
\zeta_{\max }=\frac{c_{2}+\lambda}{2 \sqrt{c_{1}}} .
\end{gathered}
$$

Combining formulas (15) and (16), the parameters $c_{1}, c_{2}$, and $\lambda$ in the nonlinear integral sliding surface (10) can be determined according to variation range of desired damping ratios or variation region of desired closed-loop poles. 
3.2. Sliding Mode Control Law Based on Nonlinear Integral Sliding Surface. According to the nonlinear integral sliding surface (10), it can be found that

$$
\begin{aligned}
\dot{s} & =\left(c_{1} e_{1}+\left(c_{2}-g\right) \dot{e}_{1}-\dot{g} e_{1}+\dot{e}_{2}\right) \\
& =c_{1} e_{1}+\left(c_{2}-g\right) \dot{e}_{1}-\dot{g} e_{1}-a_{0} x_{2}+k_{0} u+D(x)-d^{(2)} h .
\end{aligned}
$$

Let

$$
\dot{s}=-\eta \operatorname{sgn}(s)
$$

where $\eta>0$.

Let $D(x)=0$. According to formulas (17) and (18), a novel sliding mode control law can be designed as follows:

$$
\begin{gathered}
u=\frac{1}{k_{0}}\left(-c_{1} e_{1}+\left(g-c_{2}\right) \dot{e}_{1}+\dot{g} e_{1}+a_{0} x_{2}+d^{(2)} h-\eta \operatorname{sgn}(s)\right) \\
=\frac{1}{k_{0}}\left(-c_{1}\left(x_{1}-h(T, t)\right)+\left(g-c_{2}\right)\left(x_{2}-d^{(1)} h\right)\right. \\
\left.\quad+\dot{g}\left(x_{1}-h(T, t)\right)+a_{0} x_{2}+d^{(2)} h-\eta \operatorname{sgn}(s)\right) \\
=\frac{1}{k_{0}}\left(\left(\dot{g}-c_{1}\right)\left(x_{1}-h(T, t)\right)+\left(g-c_{2}\right)\left(x_{2}-d^{(1)} h\right)\right. \\
\left.\quad+a_{0} x_{2}+d^{(2)} h-\eta \operatorname{sgn}(s)\right) .
\end{gathered}
$$

Theorem 2. If the switching gain is $\eta \geq \Gamma$ in the sliding mode control law (19), then the trajectory of the closed-loop control system can reach the sliding surface (10) starting from any initial position in finite time and remain on the sliding surface.

Proof. Take the following closed-loop system Lyapunov function:

$$
V=\frac{1}{2} s^{2}
$$

Then,

$$
\begin{aligned}
\dot{V} & =s \dot{s} \\
& =s\left(c_{1} e_{1}+\left(c_{2}-g\right) \dot{e}_{1}-\dot{g} e_{1}+\dot{e}_{2}\right) \\
& =s\left(c_{1} e_{1}+\left(c_{2}-g\right) \dot{e}_{1}-\dot{g} e_{1}-a_{0} x_{2}+k_{0} u+D(x)-d^{(2)} h\right) \\
& =s(-\eta \operatorname{sgn}(s)+D(x)) \\
& =-\eta|s|+s D(x),
\end{aligned}
$$

Since $|D(x)| \leq \Gamma$, so long as $\eta$ meets the following condition:

$$
\eta \geq \Gamma
$$

It can be found that

$$
\dot{V} \leq 0
$$

TABLE 1: Fuzzy control rules.

\begin{tabular}{llllll}
\hline$s \dot{s}$ & NB & NM & ZO & PM & PB \\
\hline$\Delta \eta$ & NB & NM & ZO & PM & PB \\
\hline
\end{tabular}

Thus, according to the sliding mode control theory [30], it can be concluded that the closed-loop control system meets the sliding mode reaching condition and reaches the nonlinear integral sliding surface (10) starting from any initial position in finite time and then remains on the sliding surface.

3.3. Adaptive Adjustment of the Switching Gain. As the uncertainties of steering systems involve many factors and variables, it is difficult to determine the boundary $\Gamma$ of the system uncertainties. Even though a conservative boundary can be determined, it will make the control input do unnecessary high gain switching and aggravate the control input chattering.

In order to relax the condition $\eta \geq \Gamma$ in Theorem 2 and make the system not only meet the sliding mode reaching condition but also not to be too conservative, the fuzzy control method is utilized in the paper to adaptively adjust the switching gain $\eta$. Consequently, the control input chattering is alleviated effectively.

Since the switching gain $\eta$ is utilized to ensure that the sliding mode reaching condition is met, the fuzzy control method can be used to determine the variation $\Delta \eta$ of the $\eta$ according to the sliding mode reaching condition. The fuzzy control input is the sliding mode reaching condition $s \dot{s}$; the fuzzy control output is switching gain variation $\Delta \eta$. The fuzzy sets of fuzzy system input and output choose $\{\mathrm{NB}, \mathrm{NM}, \mathrm{ZO}, \mathrm{PM}, \mathrm{PB}\}$ and their universe of discourse is all assigned to be $\{-2,-1,0,1,2\}$. The membership functions for the fuzzy sets select the normal function as follows:

$$
\mu_{A_{i}}(x)=e^{-\left(\left(x-x_{i}\right) / \sigma_{A_{i}}\right)^{2}},
$$

where $\mu_{A_{i}}(x)$ is the membership function of the fuzzy subset $A_{i}, x_{i}$ is the central value of universe of discourse for the fuzzy subset $A_{i}$, and $\sigma_{A_{i}}$ is the parameter used to adjust the shape of the membership function.

The design experiences of fuzzy control rules in the paper are as follows: when $s \dot{s}$ is negative, $\eta$ is decreased to make the control system not too conservative; when $s \dot{s}$ is zero, $\eta$ is unchanged; when $s \dot{s}$ is positive, $\eta$ is increased in order to make the system meet the sliding mode reaching condition.

According to the above experiences, the fuzzy control rules used in the paper are designed and shown in Table 1.

The fuzzy inference adopts the Mamdani method and the defuzzification of the control output can be accomplished by the method of centre of gravity, as shown in

$$
\Delta \eta=\frac{\sum_{i=1}^{5} z_{i} \cdot \mu_{\Delta \eta}\left(z_{i}\right)}{\sum_{i=1}^{5} \mu_{\Delta \eta}\left(z_{i}\right)}
$$

where $\mu_{\Delta \eta}\left(z_{i}\right)$ is the membership function of the output and $z_{i}$ is output universe of discourse. 
Hence, the switching gain $\eta$ can be adaptively adjusted according to

$$
\dot{\eta}=k_{u} \cdot \Delta \eta,
$$

where $k_{u}$ is output scaling factor. The change of $k_{u}$ can adjust the amplitude of fuzzy control output. In practical use, trialand-error method is used to choose the value of $k_{u}$ according to experiences.

\section{Simulation Verification}

We can easily obtain that $k_{g}=50$ and $\tau=0.09$ in the mathematic model (1) by using the time domain experimental determination method [29]. In the simulation experiments, the relevant parameters are set as follows: the transient process time is $T=0.3 \mathrm{~s}$; the equivalent disturbance is $D(x)=$ $\Gamma e^{\left(-\left(\left(t-\mu_{d}\right) / \sqrt{2} \sigma_{d}\right)^{2}\right)}$, where $\mu_{d}=5, \sigma_{d}=0.5$, and $\Gamma=20$; the nonlinear integral sliding surface parameters are $c_{1}=64, c_{2}=$ 8 , and $\lambda=24$. The minimum damping ratio $\zeta_{\min }=0.5$ and the max damping ratio $\zeta_{\max }=2$ can be calculated according to formulas (15) and (16), respectively.

The step input command $\delta_{d}$ of the steering control system is set to $0.1 \mathrm{rad}$. We, respectively, choose the traditional sliding mode control method and the method proposed in the paper to control the steering system.

The traditional sliding mode control law used for comparison in the simulation verification is $u=\left(1 / k_{0}\right)\left(-c_{1}(\delta-\right.$ $\left.\left.\delta_{d}\right)-c_{2} \dot{\delta}+a_{0} \dot{\delta}-\eta_{1} \operatorname{sgn}\left(s_{1}\right)\right)$. In the sliding mode control law, $\delta$ is the actual steering angle; $\dot{\delta}$ is the steering angle rate; $s_{1}=c_{1} \int_{0}^{t}\left(\delta-\delta_{d}\right) d t+c_{2}\left(\delta-\delta_{d}\right)+\dot{\delta}$ is chosen as the traditional sliding surface; $\eta_{1} \geq \Gamma$ is the switching gain of the traditional sliding mode control law.

The nonlinear integral sliding surface-based adaptive sliding mode control law proposed in the paper is as formula (19). In the proposed sliding mode control law, the switching gain $\eta$ of the sliding mode control is adaptively adjusted by using the fuzzy control method.

Then, the step response curves of the closed-loop system are shown in Figures 3 and 4, respectively.

In the simulation experiments, the traditional sliding mode control method is firstly utilized to control the steering system. In order to speed up the system response and guarantee that the output of the steering control system reaches the desired input command in $0.3 \mathrm{~s}$, the damping ratio of the closed-loop steering system needs to be set to 0.5 by using the traditional sliding mode control law. However, it is well known that a quick response may be obtained at the cost of a large overshoot in traditional sliding mode control. Therefore, there exist overshoot phenomena in the output response, as shown in Figure 3. If the proposed method is utilized to control the steering system, the system output not only reaches the desired input command in $0.3 \mathrm{~s}$ but also is nonovershoot, as shown in Figure 4. Explanations on the results presented in the figure are that the developed nonlinear integral sliding surface (10) has monotonously increasing damping ratio characteristic. It is the characteristic that guarantees, on the one hand, that the closed-loop steering system has relatively smaller damping ratio to accelerate

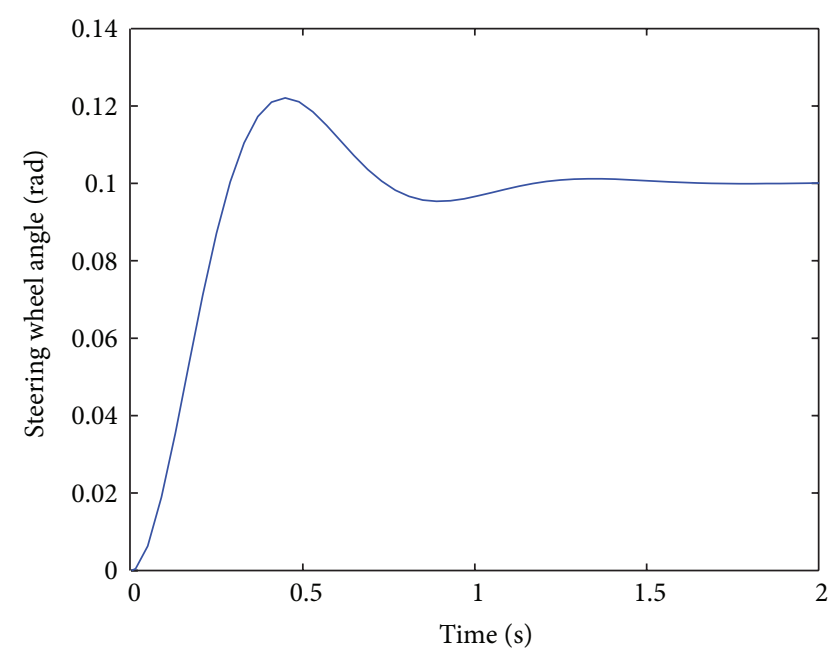

FIGURE 3: The step response curve of the traditional sliding mode control.

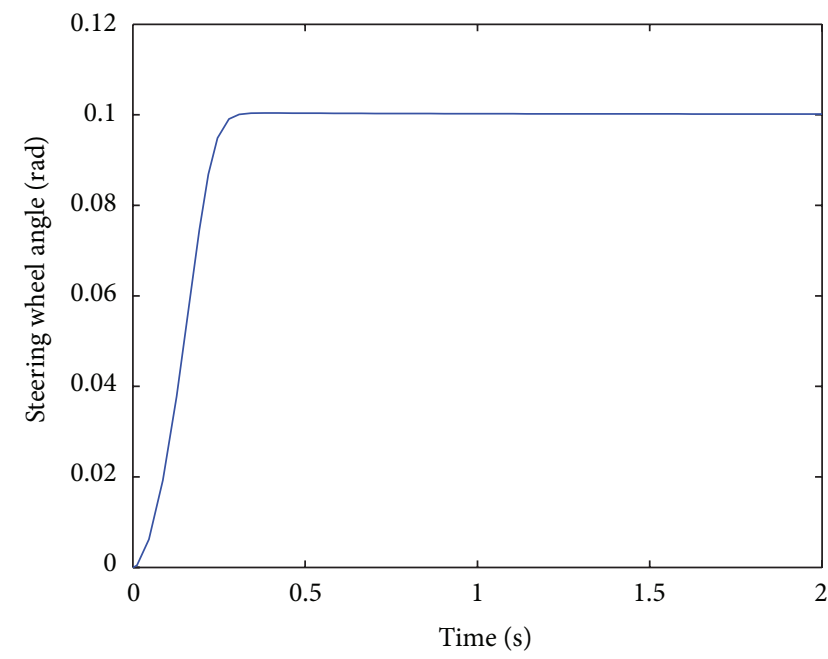

FIGURE 4: The step response curve of the proposed method in the paper.

the output response speed during the initial phase of the sliding mode movement and, on the other hand, that the closed-loop steering system has relatively bigger damping ratio to avoid system output overshoot and ensure the system stability as the output approaches the desired command.

In addition, when the steering system suffers from the disturbance $D(x)$, the fuzzy control system can accurately estimate the disturbance boundary, as shown in Figure 5. According to the estimated results, a big value of the switching gain is selected when the estimated disturbance is large; a small value of the switching gain is selected when the estimated disturbance is small. Therefore, the proposed method can adaptively adjust the switching gain $\eta$ of the sliding mode control to alleviate the chattering phenomenon and reduce the control force, as shown in Figure 6. If the traditional sliding mode control method is utilized to control the system, it is necessary to choose a sufficiently big and 


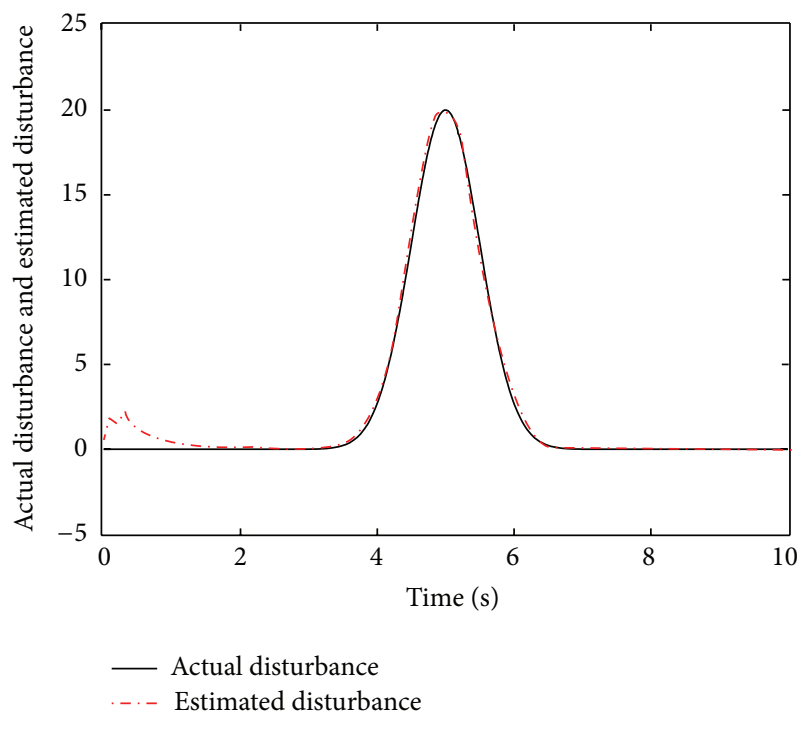

FIGURE 5: The disturbance estimation based on fuzzy control.

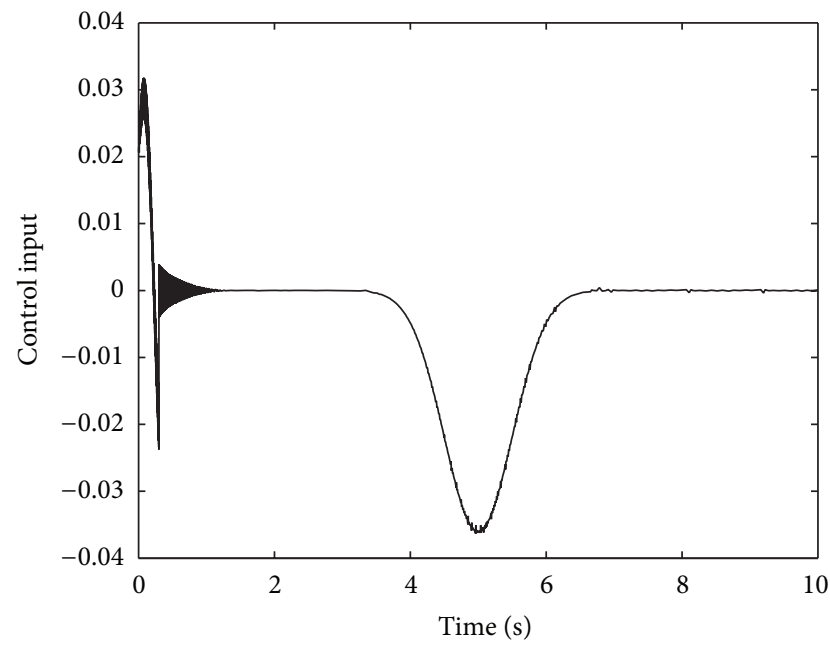

FIgURE 6: The control input of the proposed method in the paper.

fixed switching gain in order to ensure the stability of the control system. However, if a big value of switching gain is used, the control force obtained is too big and the chattering phenomenon is serious, as shown in Figure 7.

In a word, according to the simulation experiments, we can verify and conclude that the proposed method, compared to the traditional sliding mode control method, has variable damping ratio characteristic because of which the response time of the control system can be reduced without increasing the control system output overshoot. Meanwhile, the control input chattering is effectively alleviated by adaptive adjustment of the switching gain.

\section{Experimental Verification}

In order to further verify the effectiveness and superiority of the proposed method, the practical steering control experiments of a tractor are carried out in the paper. As

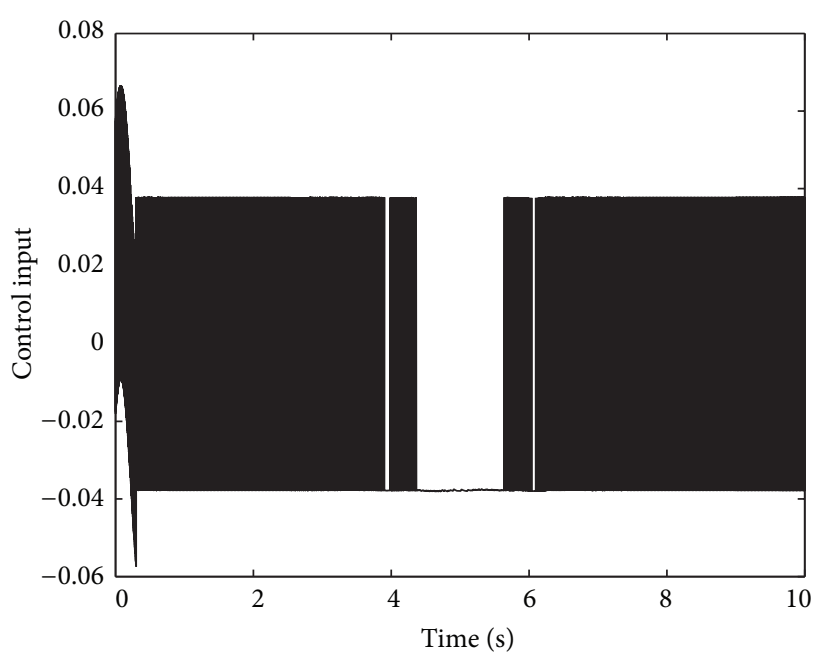

FIGURE 7: The control input of the traditional sliding mode control.

TABLE 2: The tractor parameters.

\begin{tabular}{lc}
\hline Parameter & Value \\
\hline Mass & $3115 \mathrm{~kg}$ \\
Wheelbase & $2188 \mathrm{~mm}$ \\
Turning radius & $3800 \mathrm{~mm}$ \\
Front track width & $1600 \mathrm{~mm}$ \\
Rear track width & $1730 \mathrm{~mm}$ \\
\hline
\end{tabular}

shown in Figure 8, the actual agricultural vehicle used in the experiments is the rear wheels driven tractor and its physical parameters are shown in Table 2. The mathematic model of the tractor steering system is indicated by transfer function (1). By identification experiments, we determine $k_{g}=50$ and $\tau=0.09$ in the model.

The hardware platform of the steering controller is developed based on ARM and used to run the steering control algorithm. The steering angles are measured by the steering angle measuring equipment which consists of the linkage mechanism and the encoder. The steering actuator consists of the electronic proportional valve SP08-47C, the current amplifiers, and three solenoid valves SV10-34. The steering controller completes the steering angle tracking by controlling the proportional valve flow rate and achieves automatic and manual switching of steering hydraulic loop by controlling the solenoid valve.

On the bare soil road, we, respectively, utilize the proposed adaptive sliding mode control law (19) and the widely used PID control law to carry out the tractor steering control experiments. Since the chattering phenomena of the traditional sliding mode control law are serious, the traditional sliding mode control law is not used for comparison in the steering control experiments in order to protect the steering actuator from being damaged.

In the experiments, the tractor travel speeds are $0.8 \mathrm{~m} / \mathrm{s}$ and $1.5 \mathrm{~m} / \mathrm{s}$. The control effects of the proposed method are shown in Figures 9 and 10, respectively. According to the figures, it can be concluded that the proposed method can 


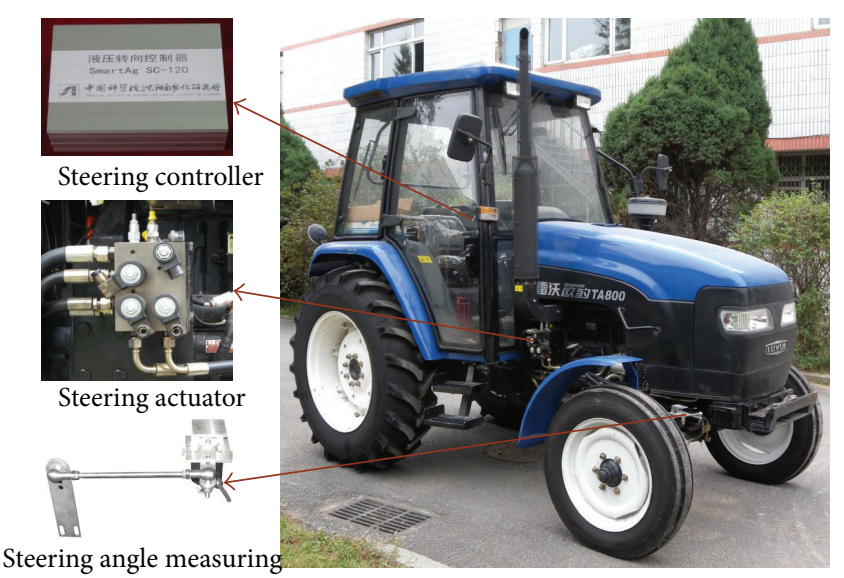

equipment

FIgURE 8: The tractor used in the experiments.

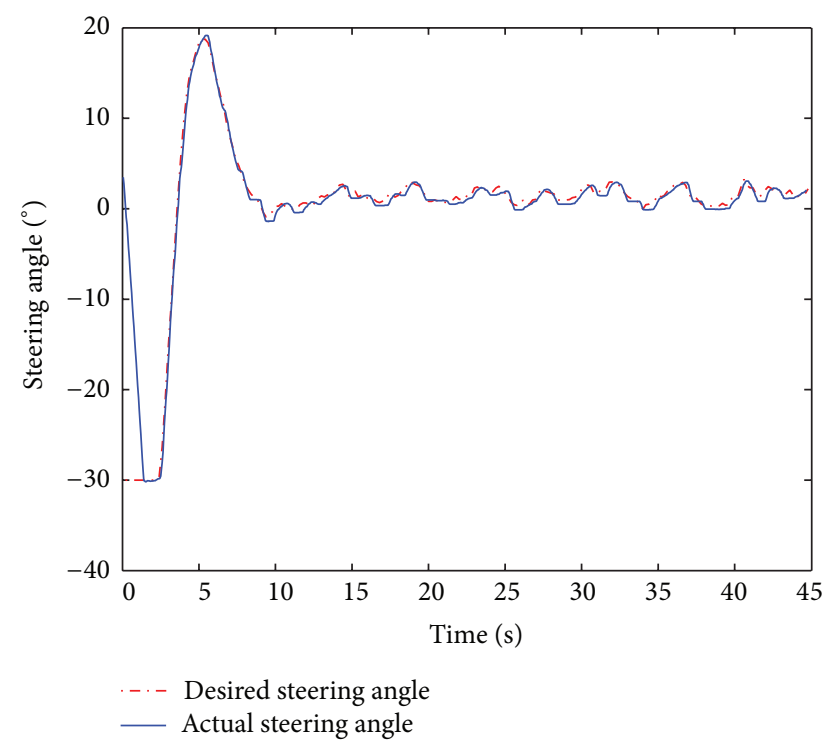

FIGURE 9: Steering control response curve based on the proposed method at a speed of $0.8 \mathrm{~m} / \mathrm{s}$.

ensure the system output to track the desired input command promptly and exactly. The analysis results of experimental data of the proposed method demonstrate that mean value of the steering angle tracking deviation is less than $0.26^{\circ}$, standard deviation is less than $0.45^{\circ}$, and max deviation is less than $1.02^{\circ}$. And these conclusions also make sense as long as travel speeds of the tractor are less than $3 \mathrm{~m} / \mathrm{s}$.

The steering control system response curves based on the PID method are shown in Figures 11 and 12. Figure 11 obviously reveals that the control system output can not track the desired input command promptly and there exists delay time. We may reduce the system damping ratio by adjusting the parameters of the PID controller and make the system response speed quicker. However, as shown in Figure 12, it will also enlarge the system output overshoot and even make the system unstable. As always necessary, one needs

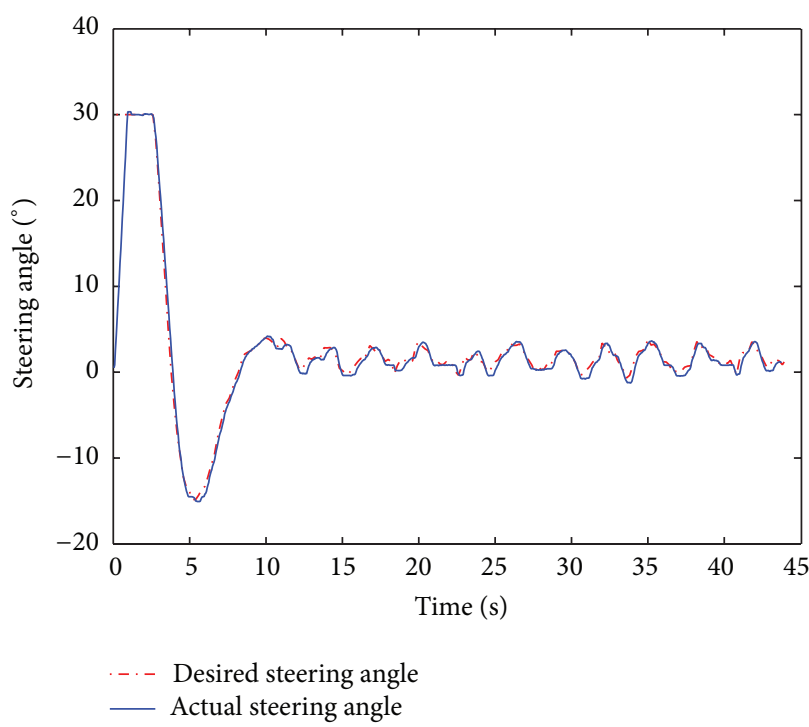

FIGURE 10: Steering control response curve based on the proposed method at a speed of $1.5 \mathrm{~m} / \mathrm{s}$.

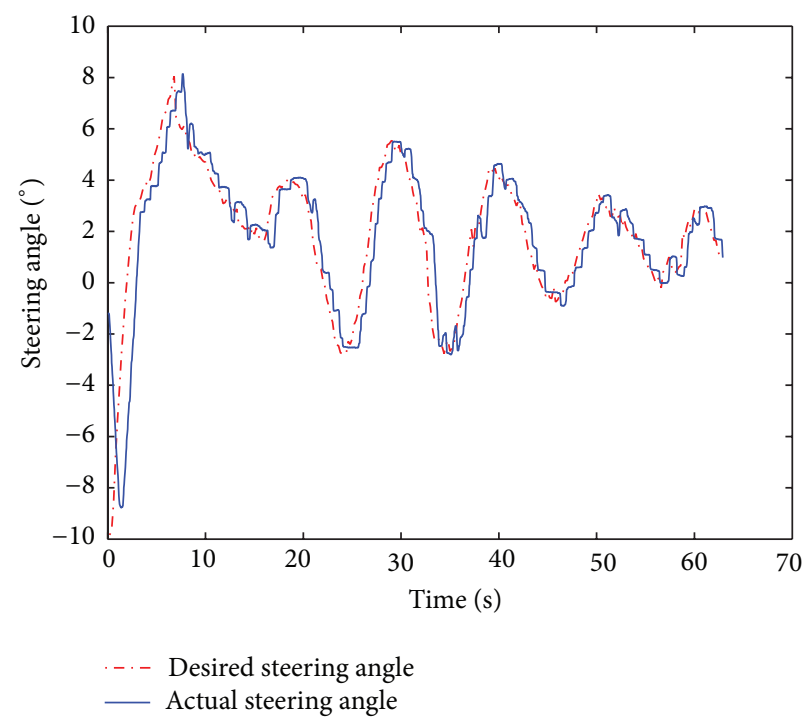

FIGURE 11: Steering control response curve based on PID method.

to tune the PID controller to obtain a suitable compromise between response time and overshoot. Meanwhile, from the experimental data perspective, the control error statistical results of the PID method are larger than those of the proposed method. Consequently, the effectiveness and the superiority of the proposed method are obviously verified by the agricultural vehicle steering control experiments.

\section{Conclusions}

In the paper, an adaptive sliding mode control method for automatic steering control of agricultural vehicles is proposed. It improves the speed and accuracy of the actual steering angle tracking the desired steering angle when 


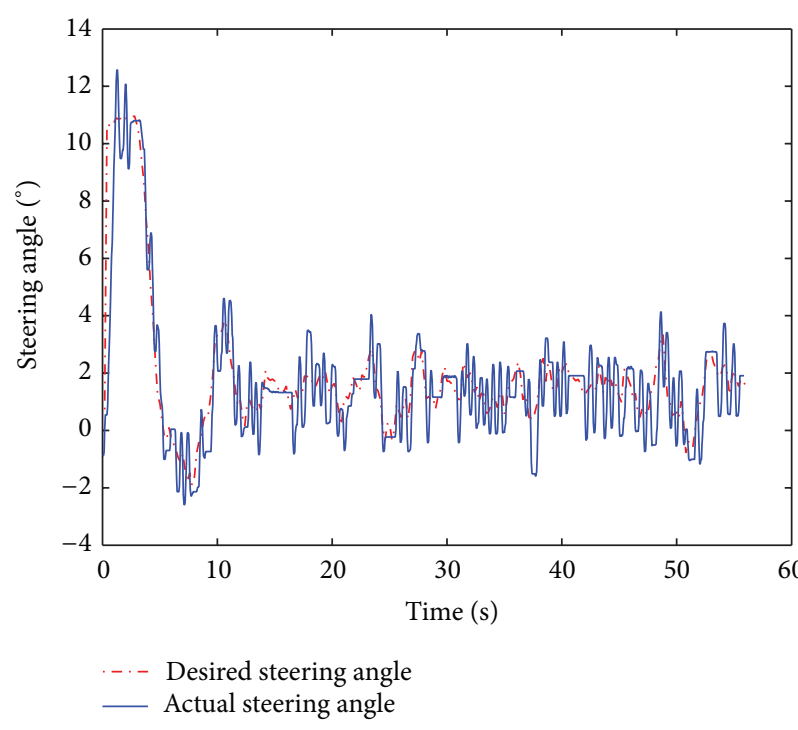

FIGURE 12: Steering control response curve based on PID method with fast response speed.

the steering system suffers from system uncertainties and external disturbances. In order to avoid producing big initial shock force to the steering actuator subsystem, we construct a transient process of the desired steering angle instead of a constant desired steering angle during each navigation control period. Then, a nonlinear integral sliding surface is developed based on the transient process. The sliding surface not only has the advantage of an ordinary integral sliding surface but also has monotonously increasing damping ratio characteristic. It is the characteristic that guarantees, on the one hand, that the closed-loop steering system has relatively smaller damping ratio to accelerate the output response speed during the initial phase of the sliding mode movement and, on the other hand, that the closed-loop steering system has relatively bigger damping ratio to avoid system output overshoot and ensure the system stability as the output approaches the desired command. In addition, in order to alleviate the control input chattering, the fuzzy control method is also introduced in the paper to adaptively adjust the switching gain.

Finally, the effectiveness and the superiority of the proposed method are verified by the simulation and agricultural vehicle experiments. The simulation experiments demonstrate that the proposed method has variable damping ratio advantage because of which the steering control system has the prompt and nonovershoot response characteristic and that the proposed method can reduce the control input chattering phenomenon. The agricultural vehicle experiments also reveal that the proposed method has better control performance than the commonly used PID method. The analysis results of experimental data of the proposed method demonstrate that the mean value of the steering angle tracking deviation is less than $0.26^{\circ}$, standard deviation is less than $0.45^{\circ}$, and the max deviation is less than $1.02^{\circ}$.

Future work includes the following two aspects: (1) improving the proposed adaptive sliding mode control method for multi-input and multioutput systems and (2) developing an exact model of the steering system using analytical methods.

\section{Conflict of Interests}

The authors declare that there is no conflict of interests regarding the publication of this paper.

\section{Acknowledgments}

This paper is supported by the National Hi-tech Research and Development Program of China (2013AA040403). The authors would like to thank Lei Gao and He Wang for their assistance in the experiments.

\section{References}

[1] J. Hu, L. Gao, H. Hu, X. Liu, X. Bai, and T. Li, "Design and development of an experiment platform for study on agricultural machinery navigation technology," International Agricultural Engineering Journal, vol. 20, no. 2, pp. 30-35, 2011.

[2] P. Jiang, Research on navigation and localization system of field working machine based on two laser source [Ph.D. thesis], Central South University, Changsha, China, 2012.

[3] H. Qiu, Navigation control for autonomous tractor guidance [Ph.D. thesis], University of Illinois at Urbana-Champaign, Urbana, Ill, USA, 2002.

[4] X. Luo, Z. Zhang, Z. Zhao, B. Chen, L. Hu, and X. Wu, "Design of DGPS navigation control system for Dongfanghong X-804 tractor," Transactions of the Chinese Society of Agricultural Engineering, vol. 25, no. 11, pp. 139-145, 2009.

[5] Z. Zhang, Automatic guidance system based on DGPS for rice transplanter [Ph.D. thesis], South China Agricultural University, Guangzhou, China, 2006.

[6] S. Lian, Research on automatic navigation control system of agricultural vehicles [M.S. thesis], Northwest A\&F University, Yangling, China, 2009.

[7] J. Zhou, Research on visual navigation for agriculture wheeled robot [Ph.D. thesis], Nanjing Agriculture University, Nanjing, China, 2003.

[8] L. Gao, J. Hu, X. Bai, T. Li, and X. Liu, "Agro-Machinery automatic navigation control system study for precision agriculture applications," in Proceedings of the International Conference on Broadcast Technology and Multimedia Communication, pp. 315322, Chongqing, China, 2010.

[9] H. Qiu, Q. Zhang, and J. F. Reid, "Fuzzy control of electrohydraulic steering systems for agricultural vehicles," Transactions of the American Society of Agricultural Engineers, vol. 44, no. 6, pp. 1397-1402, 2001.

[10] Q. Zhang, "Design of a generic fuzzy controller for electrohydraulic steering," in Proceedings of the American Control Conference, pp. 3938-3942, Arlington, Va, USA, June 2001.

[11] Q. Zhang, "A generic fuzzy electrohydraulic steering controller for off-road vehicles," Journal of Automobile Engineering, vol. 217, no. 9, pp. 791-799, 2003.

[12] F. Rovira-Más and Q. Zhang, "Fuzzy logic control of an electrohydraulic valve for auto-steering off-road vehicles," Journal of Automobile Engineering, vol. 222, no. 6, pp. 917-934, 2008. 
[13] J. B. Derrick and D. M. Bevly, "Adaptive steering control of a farm tractor with varying yaw rate properties," Journal of Field Robotics, vol. 26, no. 6-7, pp. 519-536, 2009.

[14] X. Wu, Z. Zhao, Z. Zhang, B. Chen, and L. Hu, "Development of automatic steering control system based on Dongfanghong tractor," Transactions of the Chinese Society of Agricultural Machinery, vol. 40, no. 1, pp. 1-5, 2009.

[15] X. Wu, Z. Zhao, Z. Zhang, L. Yu, L. Hu, and J. Huang, “Tractor wheels steering control system design based on dual closedloop control,' in Proceedings of the Internatiomal Agriculture Engineering Conference, pp. 64-70, Shanghai, China, 2010.

[16] W. Sun, "Adaptive sliding mode tracking control for a class of nonholonomic mechanical systems," Mathematical Problems in Engineering, vol. 2013, Article ID 734307, 9 pages, 2013.

[17] X. Yu, B. Wang, and X. Li, "Computer-controlled variable structure systems: the state-of-the-art," IEEE Transactions on Industrial Informatics, vol. 8, no. 2, pp. 197-205, 2012.

[18] Y.-J. Huang, T.-C. Kuo, and S.-H. Chang, "Adaptive slidingmode control for nonlinear systems with uncertain parameters," IEEE Transactions on Systems, Man, and Cybernetics B: Cybernetics, vol. 38, no. 2, pp. 534-539, 2008.

[19] R. Eaton, H. Pota, and J. Katupitiya, "Path tracking control of agricultural tractors with compensation for steering dynamics," in Proceedings of the 48th IEEE Conference on Decision and Control, pp. 7357-7362, Shanghai, China, December 2009.

[20] C.-F. Huang, J.-S. Lin, T.-L. Liao, C.-Y. Chen, and J.-J. Yan, "Quasi-sliding mode control of chaos in permanent magnet synchronous motor," Mathematical Problems in Engineering, vol. 2011, Article ID 964240, 10 pages, 2011.

[21] G. Bartolini, A. Ferrara, and V. I. Utkin, "Adaptive sliding mode control in discrete-time systems," Automatica, vol. 31, no. 5, pp. 769-773, 1995.

[22] Q. H. Ngo and K.-S. Hong, "Adaptive sliding mode control of container cranes," IET Control Theory and Applications, vol. 6, no. 5, pp. 662-668, 2012.

[23] J. Wang, A. B. Rad, and P. T. Chan, "Indirect adaptive fuzzy sliding mode control-part I: fuzzy switching," Fuzzy Sets and Systems, vol. 122, no. 1, pp. 21-30, 2001.

[24] W. Liu, Q. Sui, F. Zhou, and H. Xiao, "Controller design based on adaptive fuzzy sliding mode control for ship course," Information and Control, vol. 41, no. 2, pp. 136-141, 2012.

[25] Q. Zhang, D. Wu, J. F. Reid, and E. R. Benson, "Model recognition and validation for an off-road vehicle electrohydraulic steering controller," Mechatronics, vol. 12, no. 6, pp. 845-858, 2002.

[26] F. Rovira-Más, Q. Zhang, and A. C. Hansen, Mechatronics and Intelligent Systems for Off-Road Vehicles, Springer, Berlin, Germany, 2010.

[27] Y. Xia and M. Fu, Compound Control Methodology for Flight Vehicles, Springer, Berlin, Germany, 2013.

[28] N. Luo, J. Rodellar, and M. de la Sen, "Output feedback sliding mode control of base isolated structures," Journal of the Franklin Institute, vol. 337, no. 5, pp. 555-577, 2000.

[29] S. Hu, Automatic Control Theory, Science Press, Beijing, China, 2004.

[30] W. Gao, Theory and Design Method for Variable Structure Control, Science Press, Beijing, China, 1996. 


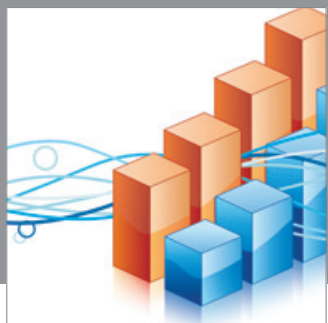

Advances in

Operations Research

mansans

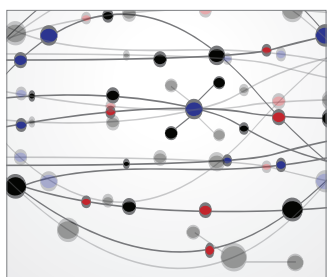

The Scientific World Journal
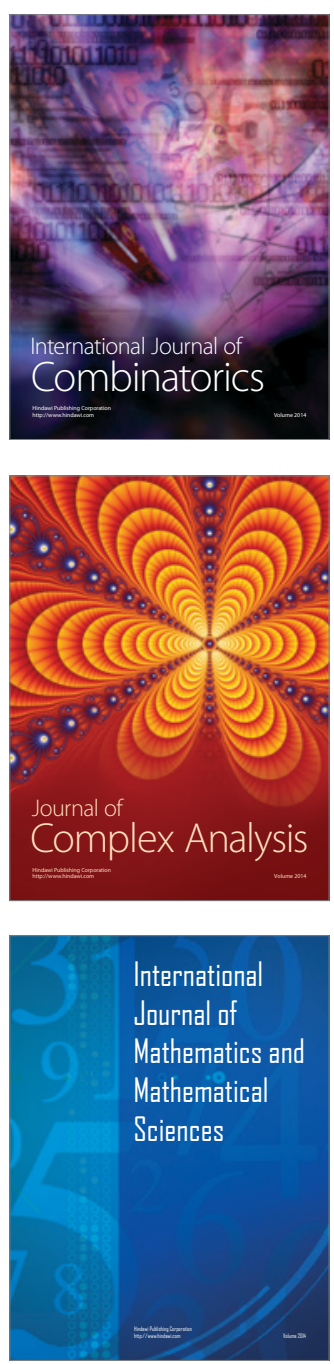
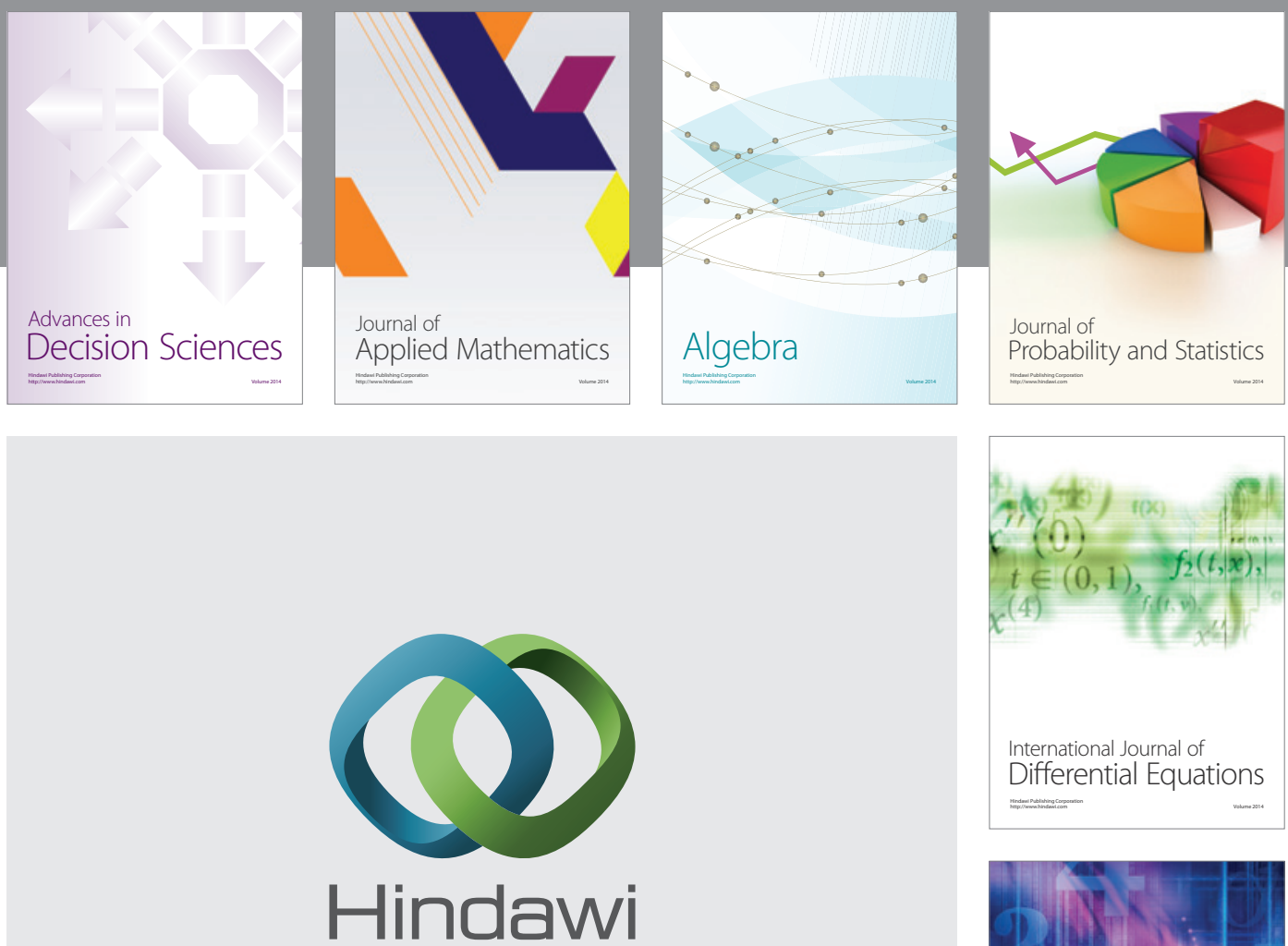

Submit your manuscripts at http://www.hindawi.com
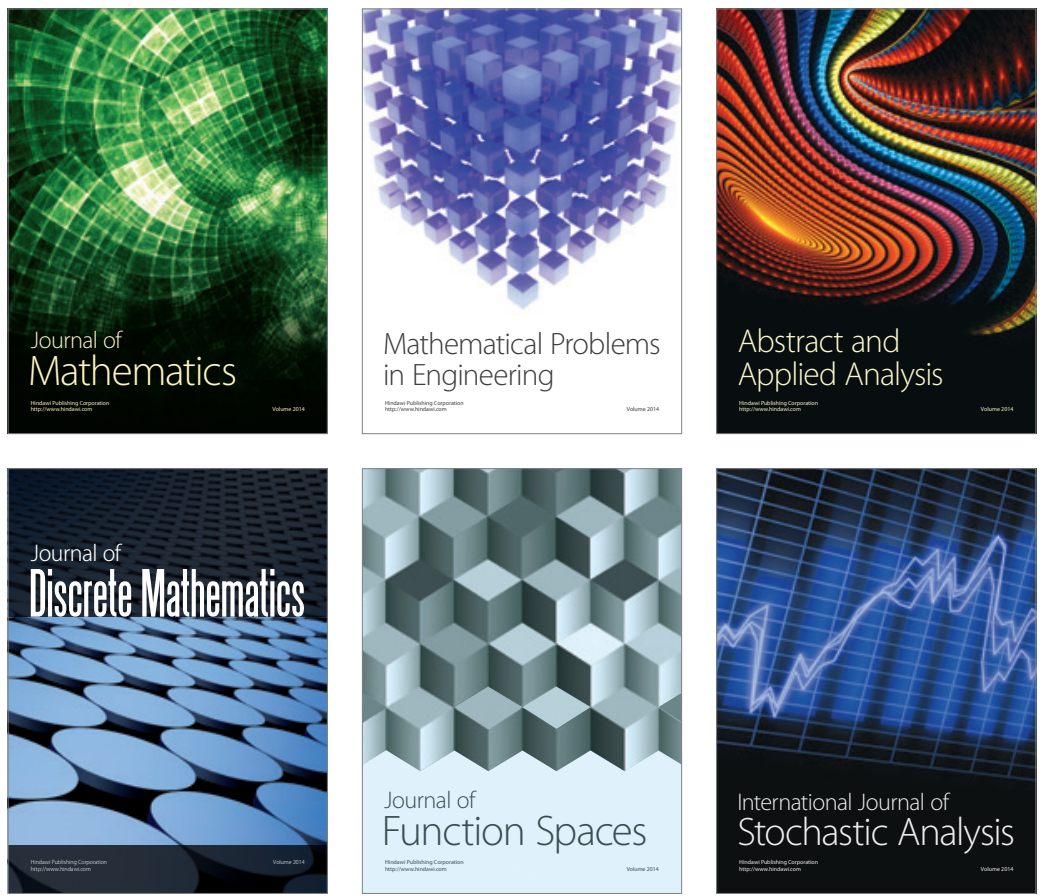

Journal of

Function Spaces

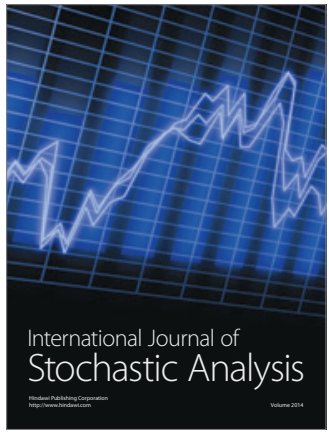

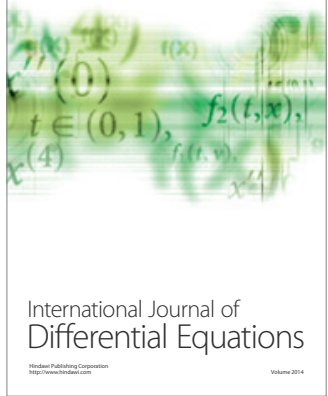
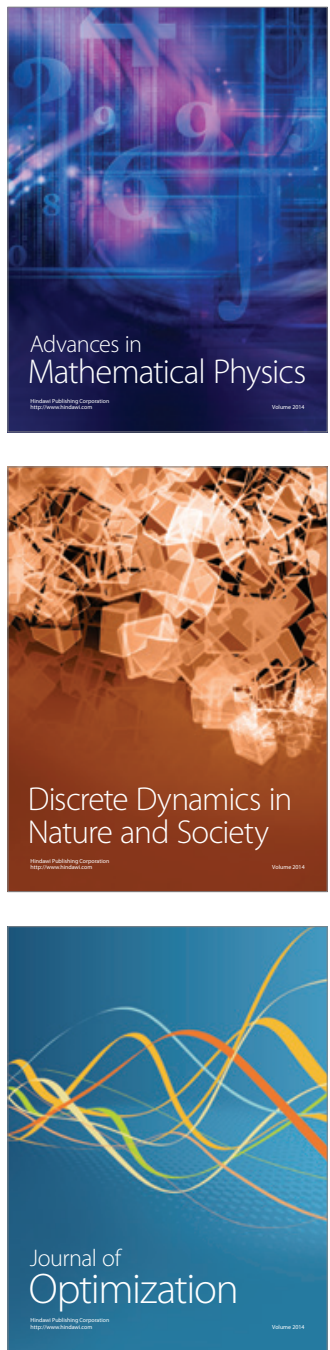